4. Other cases. The corresponding theory for a non-algebraic curve is complicated by the fact that the number of polynomials of the $n$th degree in the orthogonal system increases with $n$, but is compensatingly simplified by the observation that the representation corresponding to (2) holds for all values of $x$ and $y$, so that (except for the assumption that the domain of orthogonality is all on one side of the line) the points where the straight line meets the curve are no longer a matter of special concern. If $\left(x_{\nu}, y_{\nu}\right)$ are any $n+1$ distinct points on the line, independent polynomials of the $n$th degree orthogonal to every polynomial of lower degree with respect to the composite weight function are given by $K_{n}\left(x_{\nu}, y_{\nu}, u, v\right)$.

A similar conclusion holds for orthogonality on a two-dimensional region.

The University of Minnesota

\title{
NOTE ON AN INEQUALITY OF STEINER ${ }^{1}$
}

\section{T. RADÓ AND P. REICHELDERFER}

Let $Q$ denote the unit square $0 \leqq x, y \leqq 1$. If $f(x, y)$ be any function defined and continuous on $Q$, the relation $z=f(x, y)$ yields a continuous surface defined over $Q$. The Lebesgue area ${ }^{2}$ of this surface will be denoted by $L(f)$. Let $z=f_{1}(x, y), z=f_{2}(x, y)$ be two continuous surfaces defined over $Q$; then clearly $z=\left[f_{1}(x, y)+f_{2}(x, y)\right] / 2$ is a continuous surface defined over $Q$. The inequality of Steiner ${ }^{3}$ states that $L\left(\left[f_{1}+f_{2}\right] / 2\right) \leqq\left[L\left(f_{1}\right)+L\left(f_{2}\right)\right] / 2$. McShane ${ }^{4}$ obtained interesting and important results concerning the situation where the sign of equality holds in this relation. In this note we improve his results and, in a sense, give them a final form.

In order to emphasize and to clarify what is significant and interesting in the results of McShane and in our improvements thereon, we remind our reader of a few facts concerning the Lebesgue area. ${ }^{5}$ Given a continuous surface $z=f(x, y)$ defined over $Q$; if $L(f)$ is finite then the partial derivatives $f_{x}$ and $f_{y}$ exist almost everywhere in $Q$, the integral $\iint_{Q}\left[1+f_{x}^{2}+f_{y}^{2}\right]^{1 / 2} d x d y$ exists, and the relation

1 Presented to the Society, April 13, 1940.

${ }^{2}$ See S. Saks, Theory of the Integral, Warsaw and Lwów, 1937, chap. 5, for the facts used in this paper concerning the Lebesgue area.

${ }^{3}$ E. J. McShane, On a certain inequality of Steiner, Annals of Mathematics, (2), vol. 33 (1932), pp. 125-138.

${ }^{4}$ Loc. cit. ${ }^{3}$

${ }^{5} \mathrm{Cf}^{2}$ 
$\iint_{Q}\left[1+f_{x}^{2}+f_{y}^{2}\right]^{1 / 2} d x d y \leqq L(f)$ is valid. The sign of equality holds in this last relation if and only if $f(x, y)$ is absolutely continuous in the sense of Tonelli. Next, if a sequence of continuous functions $f_{n}(x, y)$ converges uniformly on $Q$ to $f(x, y)$, then it is always true that $\lim \inf _{n \rightarrow \infty} L\left(f_{n}\right) \geqq L(f)$, but the sign of equality holds in this last relation only if the sequence $f_{n}(x, y)$ is chosen with extreme care.

Suppose $z=f_{1}(x, y), z=f_{2}(x, y)$ are two continuous surfaces defined over $Q$ such that $L\left(\left[f_{1}+f_{2}\right] / 2\right)=\left[L\left(f_{1}\right)+L\left(f_{2}\right)\right] / 2$. If we assume, for the moment, that both $f_{1}(x, y)$ and $f_{2}(x, y)$ are absolutely continuous in the sense of Tonelli, then so is $\left[f_{1}(x, y)+f_{2}(x, y)\right] / 2$, and the preceding equality is equivalent to

$$
\begin{aligned}
\iint_{Q}\{ & \frac{\left[1+f_{1 x}^{2}+f_{1 y}^{2}\right]^{1 / 2}+\left[1+f_{2 x}^{2}+f_{2 y}^{2}\right]^{1 / 2}}{2}- \\
& \left.-\left[1+\left(\frac{f_{1 x}+f_{2 x}}{2}\right)^{2}+\left(\frac{f_{1 y}+f_{2 y}}{2}\right)^{2}\right]^{1 / 2}\right\} d x d y=0 .
\end{aligned}
$$

Using the inequality of Schwartz one sees that the integrand in (1) is never negative; thus the integrand is equal to zero almost everywhere in $Q$, which implies that $f_{1 x}=f_{2 x}, f_{1 y}=f_{2 y}$ almost everywhere in $Q$-that is, $\left[\left(f_{1 x}-f_{2 x}\right)^{2}+\left(f_{1 y}-f_{2 y}\right)^{2}\right]^{1 / 2}=0$ almost everywhere in $Q{ }^{6}$

Now suppose we discard the assumptions that $f_{1}$ and $f_{2}$ are absolutely continuous in the sense of Tonelli; then, of course, the preceding reasoning yields no result. Still McShane proves that $\left[\left(f_{1 x}-f_{2 x}\right)^{2}+\left(f_{1 y}-f_{2 y}\right)^{2}\right]^{1 / 2}=0$ almost everywhere in $Q$. Indeed, he proves a sharper result to the effect that, when $\left[L\left(f_{1}\right)+L\left(f_{2}\right)\right] / 2$ $-L\left(\left[f_{1}+f_{2}\right] / 2\right)$ is small, then $\left[\left(f_{1 x}-f_{2 x}\right)^{2}+\left(f_{1 y}-f_{2 y}\right)^{2}\right]^{1 / 2}$ is small in measure, so to speak-quite exactly, he establishes a contrapositive statement: If the functions $f_{1}(x, y), f_{2}(x, y)$ are defined and continuous on a square $Q$ and there exist positive numbers $G, \epsilon, \delta$ such that

(1) $L\left(f_{1}\right) \leqq G, L\left(f_{2}\right) \leqq G$;

(2) $\left[\left(f_{1 x}-f_{2 x}\right)^{2}+\left(f_{1 y}-f_{2 y}\right)^{2}\right]^{1 / 2} \geqq \epsilon$ on a set of measure at least equal to $\delta$;

then

$$
L\left(\left[f_{1}+f_{2}\right] / 2\right) \leqq\left[L\left(f_{1}\right)+L\left(f_{2}\right)\right] / 2-\mu(G, \epsilon, \delta),
$$

where $\mu$ is a positive number whose value depends only on $G, \epsilon$, and $\delta$. We improve this result by replacing smallness with respect to

${ }^{6}$ From this it follows (loc. cit., ${ }^{3}$ Theorem I) that $f_{1}-f_{2}$ is constant on $Q$, but we shall not be concerned with this fact.

${ }^{7}$ Loc. cit., ${ }^{3}$ Theorem IV. 
measure by smallness with respect to exponent. First, we show that, if $f_{1}(x, y)$ and $f_{2}(x, y)$ are two continuous functions defined on the square $Q$ for which $L\left(f_{1}\right)$ and $L\left(f_{2}\right)$ are both finite, then

$$
\begin{aligned}
& \left\{\iint_{Q}\left[\left(f_{1 x}-f_{2 x}\right)^{2}+\left(f_{1 y}-f_{2 y}\right)^{2}\right]^{1 / 4} d x d y\right\}^{4} \\
& \quad \leqq\left[L\left(f_{1}\right)+L\left(f_{2}\right)\right]^{3}\left[\frac{L\left(f_{1}\right)+L\left(f_{2}\right)}{2}-L\left(\frac{f_{1}+f_{2}}{2}\right)\right] .
\end{aligned}
$$

Since the Hölder inequality reveals

$$
\left\{\iint_{Q}\left[\left(f_{1 x}-f_{2 x}\right)^{2}+\left(f_{1 y}-f_{2 y}\right)^{2}\right]^{\lambda / 2} d x d y\right\}^{2 / \lambda}
$$

to be a non-decreasing function of $\lambda$, it follows that, for every exponent $\lambda$ satisfying $0<\lambda \leqq 1 / 2$, it is true that

$$
\begin{aligned}
&\left\{\iint_{Q}\left[\left(f_{1 x}-f_{2 x}\right)^{2}+\left(f_{1 y}-f_{2 y}\right)^{2}\right]^{\lambda / 2} d x d y\right\}^{2 / \lambda} \\
& \leqq\left[L\left(f_{1}\right)+L\left(f_{2}\right)\right]^{3}\left[\frac{L\left(f_{1}\right)+L\left(f_{2}\right)}{2}-L\left(\frac{f_{1}+f_{2}}{2}\right)\right] .
\end{aligned}
$$

Next, we prove that, under the same assumptions, it follows that

$$
\begin{aligned}
& \left\{\iint_{Q}\left[\left(f_{1 x}-f_{2 x}\right)^{2}+\left(f_{1 y}-f_{2 y}\right)^{2}\right]^{\lambda / 2} d x d y\right\}^{2 / \lambda} \\
& \leqq\left[L\left(f_{1}\right)+L\left(f_{2}\right)\right]^{(1+\lambda) / \lambda}\left[\frac{L\left(f_{1}\right)+L\left(f_{2}\right)}{2}-L\left(\frac{f_{1}+f_{2}}{2}\right)\right]^{(1-\lambda) / \lambda}
\end{aligned}
$$

for every exponent $\lambda$ satisfying $1 / 2 \leqq \lambda \leqq 1$. The reader will observe that inequality (4) reduces to (2) for $\lambda=1 / 2$.

Let, now, $f_{n}(x, y)$ be a sequence of continuous functions converging uniformly on $Q$ to $f(x, y)$; assume that $L\left(f_{n}\right)$ and $L(f)$ are finite, and that $L\left(f_{n}\right)$ converges to $L(f)$. Under these hypotheses McShane proves that $\left[\left(f_{n x}-f_{x}\right)^{2}+\left(f_{n y}-f_{y}\right)^{2}\right]^{1 / 2}$ converges to zero in measure. ${ }^{8}$ We improve this result by showing that under these hypotheses it is true that

$$
\lim _{n \rightarrow \infty} \iint_{Q}\left[\left(f_{n x}-f_{x}\right)^{2}+\left(f_{n y}-f_{y}\right)^{2}\right]^{\lambda / 2} d x d y=0
$$

for every exponent $\lambda$ satisfying $0<\lambda<1$. Since McShane has shown

\footnotetext{
${ }^{8}$ Loc. cit., ${ }^{3}$ Theorem V.
} 
that the relation (5) is not generally true for the exponent $\lambda=1,{ }^{9}$ our result is the best obtainable under the given hypotheses.

In fact, we shall give two proofs for relation (5). One proof will reveal this result as an immediate consequence of inequalities (3) and (4); the other will depend upon a general lemma concerning the Lebesgue integral.

We proceed presently to the proof of inequality (2). Given two continuous functions $f_{1}(x, y), f_{2}(x, y)$ defined on $Q$ for which $L\left(f_{1}\right)$, $L\left(f_{2}\right)$ are both finite; then $f_{1 x}, f_{1 y} ; f_{2 x}, f_{2 y}$ exist almost everywhere in $Q$. Set

$$
\begin{array}{rlrl}
u & =\left\lfloor\left(f_{1 x}-f_{2 x}\right)^{2}+\left(f_{1 y}-f_{2 y}\right)^{2}\right]^{1 / 2} ; \quad v=1+f_{1 x} f_{2 x}+f_{1 y} f_{2 y} ; \\
w_{1} & =\left[1+f_{1 x}^{2}+f_{1 y}^{2}\right]^{1 / 2} ; & w_{2}=\left[1+f_{2 x}^{2}+f_{2 y}^{2}\right]^{1 / 2} ; \\
w & =\left[1+\left(\left[f_{1 x}+f_{2 x}\right] / 2\right)^{2}+\left(\left[f_{1 y}+f_{2 y}\right] / 2\right)^{2}\right]^{1 / 2} ; \\
\alpha & =\left(w_{1}+w_{2}\right) / 2-w ; \quad \beta=\left(w_{1}+w_{2}\right) / 2+w ; \\
\gamma & =w_{1} w_{2}-v ; \quad \delta=w_{1} w_{2}+v .
\end{array}
$$

The reader will easily verify the following identities:

$$
\begin{array}{ll}
\alpha+\beta=w_{1}+w_{2}, & \alpha \cdot \beta=\gamma / 2, \\
\gamma+\delta=2 w_{1} w_{2}, & \gamma \cdot \delta=u^{2}+\left(f_{1 x} f_{2 y}-f_{2 x} f_{1 y}\right)^{2} .
\end{array}
$$

Now ( 7 ) implies that $\gamma \geqq 0, \delta \geqq 0$, whence it follows that $\alpha \cdot \beta \geqq 0$, and that

$$
\delta \leqq 2 w_{1} w_{2} \leqq\left(w_{1}+w_{2}\right)^{2} / 2 \text {. }
$$

In view of these relations (6) implies that $\alpha \geqq 0, \beta \geqq 0$; hence $\beta \leqq w_{1}+w_{2}$. So the reader sees that

$$
u^{2} \leqq \gamma \delta=2 \alpha \beta \delta \leqq\left(w_{1}+w_{2}\right)^{3}\left\{\left(w_{1}+w_{2}\right) / 2-w\right\} .
$$

As we observed above, it is true that

$$
\iint_{Q} w_{i} d x d y \leqq L\left(f_{i}\right), \quad i=1,2 .
$$

A reasoning of $\mathrm{McShane}^{10}$ shows that

$$
\begin{aligned}
\iint_{Q}\left\{\left(w_{1}+w_{2}\right) / 2-w\right\} & d x d y \\
& \leqq\left[L\left(f_{1}\right)+L\left(f_{2}\right)\right] / 2-L\left(\left[f_{1}+f_{2}\right] / 2\right) .
\end{aligned}
$$

Using the Hölder inequality the reader will speedily verify that inequality (2) follows from (8), (9), and (10).

${ }^{9}$ Loc. cit., ${ }^{3}$ Theorem VI.

${ }^{10}$ Loc. cit., ${ }^{3}$ p. 129. 
We proceed next to establish inequality (4). It follows by the triangle inequality that $u \leqq w_{1}+w_{2}$; hence we have from (9),

$$
\iint_{Q}\left[\left(f_{1 x}-f_{2 x}\right)^{2}+\left(f_{1 y}-f_{2 y}\right)^{2}\right]^{1 / 2} d x d y \leqq L\left(f_{1}\right)+L\left(f_{2}\right) .
$$

Suppose that $\lambda_{2}$ is a positive number and $F(x, y)$ is a non-negative function such that $F^{\lambda_{2}}$ is summable on $Q$. If $\lambda_{1}$ be any positive number less than $\lambda_{2}$, it follows by the Hölder inequality that $F^{\lambda_{1}}$ is also summable on $Q$. Now every number $\lambda$ satisfying $\lambda_{1} \leqq \lambda \leqq \lambda_{2}$ may be written in the form $\lambda=\alpha_{1} \lambda_{1}+\alpha_{2} \lambda_{2}$, where $0 \leqq \alpha_{1}, \alpha_{2} \leqq 1, \alpha_{1}+\alpha_{2}=1$. From the Hölder inequality we have

$$
\iint_{Q} F^{\lambda} d x d y \leqq\left[\iint_{Q} F^{\lambda_{1}} d x d y\right]^{\alpha_{1}}\left[\iint_{Q} F^{\lambda_{2}} d x d y\right]^{\alpha_{2}} .
$$

The reader will verify that (4) follows from (2), (11), and (12) with $F=u, \lambda_{1}=1 / 2, \lambda_{2}=1$.

Now let $f_{n}(x, y)$ be a sequence of continuous functions converging uniformly on $Q$ to $f(x, y)$; assume that $L\left(f_{n}\right)$ and $L(f)$ are finite, and that $L\left(f_{n}\right)$ converges to $L(f)$. We first show that

$$
\lim _{n \rightarrow \infty}\left[\frac{L\left(f_{n}\right)+L(f)}{2}-L\left(\frac{f_{n}+f}{2}\right)\right]=0 .
$$

Since $\left(f_{n}+f\right) / 2$ converges uniformly to $f$ on $Q$, it follows that

$$
\liminf _{n \rightarrow \infty} L\left(\frac{f_{n}+f}{2}\right) \geqq L(f) .
$$

But $\lim _{n \rightarrow \infty}\left[L\left(f_{n}\right)+L(f)\right] / 2=L(f)$, and by the inequality of Steiner it follows that $L\left(\left[f_{n}+f\right] / 2\right) \leqq\left[L\left(f_{n}\right)+L(f)\right] / 2$. Thus

$$
\limsup _{n \rightarrow \infty} L\left(\frac{f_{n}+f}{2}\right) \leqq L(f) .
$$

From (14) and (15), the assertion in (13) follows.

Now the inequality (3) shows that, for $0<\lambda \leqq 1 / 2$, it is true that

$$
\begin{aligned}
& \iint_{Q}\left[\left(f_{n x}-f_{x}\right)^{2}+\left(f_{n y}-f_{y}\right)^{2}\right]^{\lambda / 2} d x d y \\
& \quad \leqq\left[L\left(f_{n}\right)+L(f)\right]^{3 \lambda / 2}\left[\frac{L\left(f_{n}\right)+L(f)}{2}-L\left(\frac{f_{n}+f}{2}\right)\right]^{\lambda / 2},
\end{aligned}
$$

while inequality (4) shows that, for $1 / 2 \leqq \lambda \leqq 1$, it is true that 


$$
\begin{aligned}
& \iint_{Q}\left[\left(f_{n x}-f_{x}\right)^{2}+\left(f_{n y}-f_{y}\right)^{2}\right]^{\lambda / 2} d x d y \\
& \quad \leqq\left[L\left(f_{n}\right)+L(f)\right]^{(1+\lambda) / 2}\left[\frac{L\left(f_{n}\right)+L(f)}{2}-L\left(\frac{f_{n}+f}{2}\right)\right]^{(1-\lambda) / 2} .
\end{aligned}
$$

From (13), (16), (17) the relation (5) clearly follows.

In order to give a second proof of relation (5) we use the following lemma. ${ }^{11}$ Let $F_{n}(x, y)$ be a sequence of non-negative functions defined on $Q$ for which there exists a positive number $\lambda_{0}$ such that (1) the functions $F_{n}$ converge to zero in measure; (2) the functions $F_{n}^{\lambda_{0}}$ are summable on $Q$; (3) the integrals $\iint_{Q} F_{n}^{\lambda_{0}} d x d y$ are uniformly bounded by a positive number $M$. Then, for every positive number $\lambda$ less than $\lambda_{0}$, it is true that $\lim _{n \rightarrow \infty} \iint_{Q} F_{n}^{\lambda} d x d y=0$.

Proof. Given $\epsilon>0$, since $F_{n}$ converges to zero in measure there exists a positive integer $n(\epsilon)$ such that, for every $n>n(\epsilon)$ the set $E_{n}(\epsilon)$ of points $(x, y)$ in $Q$ for which $F_{n}(x, y) \geqq \epsilon^{1 / \lambda}$ is of measure less than $\epsilon^{\lambda_{0} /\left(\lambda_{0}-\lambda\right)} \cdot M^{-\lambda /\left(\lambda_{0}-\lambda\right)}$. Now obviously

$$
\iint_{Q} F_{n}^{\lambda} d x d y=\iint_{Q-E_{n}(\epsilon)} F_{n}^{\lambda} d x d y+\iint_{E_{n}(\epsilon)} F_{n}^{\lambda} d x d y
$$

On $Q-E_{n}(\epsilon)$ it is clear that $F_{n}^{\lambda}(x, y) \leqq \epsilon$; hence

$$
\iint_{Q-E_{n}(\epsilon)} F_{n}^{\lambda} d x d y \leqq \epsilon .
$$

Using the Hölder inequality, we have, for $n>n(\epsilon)$,

$$
\begin{aligned}
\iint_{E_{n}(\epsilon)} F_{n}^{\lambda} d x d y \\
\qquad\left[\iint_{E_{n}(\epsilon)} F_{n}^{\lambda_{0}} d x d y\right]^{\lambda / \lambda_{0}}\left[\iint_{E_{n}(\epsilon)} d x d y\right]^{\left(\lambda_{0}-\lambda\right) / \lambda_{0}}<\epsilon .
\end{aligned}
$$

Since $\epsilon$ is arbitrary, the lemma follows immediately from (18), (19), (20).

Now let $f_{n}(x, y)$ be a sequence of continuous functions converging uniformly on $Q$ to a function $f(x, y)$; assume that $L\left(f_{n}\right)$ and $L(f)$ are finite, and that $\lim _{n \rightarrow \infty} L\left(f_{n}\right)=L(f)$. Consider the sequence of functions $F_{n}(x, y)=\left[\left(f_{n x}-f_{x}\right)^{2}+\left(f_{n y}-f_{y}\right)^{2}\right]^{1 / 2}$. In view of the results of McShane

${ }^{11}$ This lemma is an immediate corollary of certain important results of F. Riesz, Untersuchungen über Systeme integrierbarer Funktionen, Mathematische Annalen, vol. 69 (1910), pp. 449-497. We give a direct proof for the convenience of the reader. 
stated above, it is clear that this sequence of functions satisfies the three conditions of the preceding lemma with $\lambda_{0}=1$. Thus, for every exponent $\lambda$ satisfying $0<\lambda<1$ it is true that

$$
\lim _{n \rightarrow \infty} \iint_{Q}\left[\left(f_{n x}-f_{x}\right)^{2}+\left(f_{n y}-f_{y}\right)^{2}\right]^{\lambda / 2} d x d y=0 .
$$

So we have a second proof of our result (5).

The Ohio State University 